\title{
Enhancing the Student Learning in Engineering Education with the Virtual Reality Experiences
}

\author{
*1 Ali Sheharyar and ${ }^{1}$ Othmane Bouhali \\ ${ }^{1}$ Research Computing, Texas A\&M University at Qatar, Doha, Qatar
}

\begin{abstract}
Virtual reality (VR), defined as three-dimensional immersive and realistic environment, is pushing its way into becoming the mainstream in many aspects of daily life. It promises to provide more engaging and immersive experiences in several areas, including training, safety and education. In the education sector particularly, it can increase student engagement, provide active and constructive learning, and provide a platform for visualizing complex concepts concretely. Texas A\&M University at Qatar (TAMUQ), one of six American university campuses in Qatar, was one of the first in adopting the virtual reality technology in the education and research in the region. It acquired its first immersive VR system in 2008 and the second more sophisticated system in 2016. It organizes an annual project competition to promote the use of virtual reality and 3D visualization in the academic community. This paper describes the salient VR projects completed in last few years at TAMUQ.
\end{abstract}

Key words: virtual reality, education, engineering, visualization, simulation

\section{Introduction}

It has been realized since decades that VR has the potential to revolutionize the education and enhance the learning outcome. It has been believed that VR could be used for simulation-based education, where students could learn new skills by practicing repeatedly and safely with the assumption that they have access to expensive and specialized environments. Despite the prospects, the VR technology had been limited to dedicated training simulators for military, pilots and surgeons. It never became ready to be applied in education and training at large [1]. However, with the release of first consumer-priced Head Mounted Display (HMD), Oculus Rift ${ }^{\circledR}$ [2] the VR technology started becoming accessible to the wider public for research and education as well.

The term "Virtual Reality" (VR) has been used back in the 60s. It has evolved and become more and more similar to the real world. For decades, it has been a luxury that only military, large academic and research centers could afford. Now, it has become affordable and sophisticated and is getting more attention. The market for VR and augmented reality (AR) is expected to grow by an average of 50 percent annually over the next five years [3]. In 2022, a total of 121 million units will be sold, with a value of $\$ 9.9$ billion [3]. The consumer HMDs such as Oculus Rift ${ }^{\circledR}[2]$ and HTC Vive ${ }^{\circledR}[4]$ have ignited a new wave of growth that has helped broaden the appeal of virtual reality. These low-cost VR devices have broadened the appeal of virtual reality, particularly in education [3].

The application of VR in education can be considered as one of the natural evolutions of computer-

*Corresponding author: Address: Texas A\&M University at Qatar c/o Qatar Foundation, P. O. Box 23874, Doha, Qatar. E-mail address: ali.sheharyar@qatar.tamu.edu, Phone: +974-44230229 
assisted instruction (CAI) or computer-based training (CBT) [5]. Numerous studies have been conducted on its applications and effectiveness in education [6]. It offers many advantages that are shared in many ways to the advantages of using a computer or interactive simulation, particularly with 3-D models. One of its major advantages is that it grabs and holds the attention of students and is highly motivating [7]. It can expose learners to challenging scenarios and allow them to practice new skills that enables repetition, correction and safe failures [1].

Texas A\&M University at Qatar (TAMUQ) campus is one of the six American university campuses established in the Education City, Doha, Qatar. It currently offers four ABET-accredited engineering degrees including Petroleum, Electrical, Mechanical and Chemical engineering. It has a sophisticated traditional VR system since 2008 as well as recently started developing for the modern head-mounted VR devices such as Oculus Rift ${ }^{\circledR}[2]$, and HTC Vive ${ }^{\circledR}[4]$ etc. In order to promote the use of virtual reality and 3D visualization among students, faculty and researchers, it organizes an annual competition for the development of 3D scientific and virtual reality applications. The competition is usually open to other universities and government institutions in the country [8].

In nearly ten years, there have been many exciting educational and research projects developed using the TAMUQ VR system. For instance, Dr. Anuj Gupta, a petroleum engineering faculty, used the immersive VR system to teach students the pore structures of core samples, the subsurface reservoirs and well systems [9, 10]. Adnan et al. [11] used VR to visualize the impact of wireless signals on the human brain and used this project to assist in the engineering education. In a project in collaboration with Qatar Museum Authority, Tetlow et al. [12] visualized the sea-level changes and its effects on patterns of human occupation from the data collected from north-western Qatar. Abdalla et al. [13], studied the three-dimensional flow of hydrogen and carbon in a vortex solar reactor.

In this paper, we start by giving an overview of the 3D technologies installed and used in TAMUQ university. We then highlight few projects where we have used VR as a tool to improve the learning experience. We conclude by our future plans.

\section{Overview: Virtual Reality Technologies}

There are various approaches to implementing the VR systems. The head mounted displays (HMD) and CAVE approaches are two of the best known. This section briefly describes these systems and compare them.

\subsection{Cave Automatic Virtual Environment (CAVE)}

The CAVE systems [14] allow a group of users to experience the virtual reality application at the same place simultaneously. It is usually a small room or a cubicle with at least three surfaces (walls, floor and/or ceiling) acting as projection planes. In a typical 4-sided system, the walls are rearprojected screens so that the participants in the CAVE do not cast shadows. The floor has a downprojected screen so shadows are cast but the projector is positioned at angle at top of the front screen to have the user shadows fall mainly behind them. To project at all six sides of the cube 
including both floor and ceiling, the rear-projection is used at all sides but it requires space at top and bottom. Usually, two building stories are reserved for such configurations.

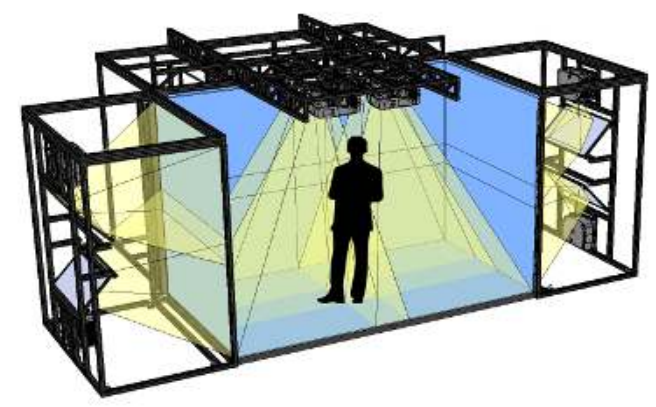

Figure 1. An example of a CAVE virtual reality system [15]

The user would wear a pair of shutter glasses in order to perceive 3D stereo vision. The projectors are synced to the shutter glasses to produce images that are for the corresponding eye at the correct moment. The images are generated by computers in coordination with a motion tracker attached to the viewer's shutter glasses. The head position and orientation are factored in to how the rendered images are displayed on the projection surfaces. As a result, the viewer is able to explore the virtual world by moving around the space and interact with the virtual objects with devices such as flystick, a wand-like device. Multiple viewers often share virtual experiences and easily carry on discussions, enabling them to exchange insights. One user is considered the active viewer, controlling the stereo projection reference point, while several additional users can simultaneously participate as passive viewers. Figure 1 shows a sample four-sided CAVE system.

\subsection{Head-Mounted Display (HMD)}

In HMD VR, a user wears a device which hosts a separate display for each eye. The displays stream data, images and other information in front of the user's eyes. The device covers the entire field of vision of the user and provides a view into the virtual world. Consequently, users cannot see their hands nor the devices that they are controlling. After the release of Oculus Rift DK1 in 2015, the HMDs became cheaper, sophisticated and accessible to the consumers. In this section, we will briefly describe only the most recent HMD VR devices.

There are two types of HMDs: 1) Discrete HMD, and 2) Mobile HMD. Discrete HMD, also known as Tethered HMD is connected to a PC through cable that generates the images for the displays. Hata! Başvuru kaynağı bulunamadı. shows a discrete HMD, an Oculus Rift ${ }^{\circledR}$ [2], along with its hand-held motion controllers. On the other hand, Mobile HMD, also known as Standalone VR HMD requires a smartphone to work. It uses smartphone display, tracking and processing to function. Figure 2 shows picture of an Oculus Rift (a discrete HMD) and Samsung Gear ${ }^{\circledR}$ VR headset with a Samsung Galaxy ${ }^{\circledR}$ smartphone attached (a mobile VR HMD). 

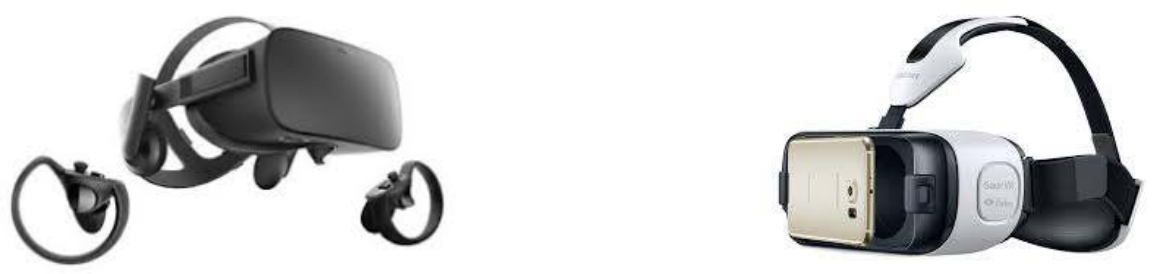

Figure 2. Oculus Rift (left), Samsung Gear VR (right)

The HMDs have been getting much more attention nowadays. They are indeed capable of transporting users to different environments and immersing them realistically. However, there are certain limitations of HMDs. One major limitation is that it completely blocks out the users from the surroundings, unable to see their own bodies, hands, feet, or the real-world obstacles around them. Many of novice users who try VR can run into walls, furniture or other colleagues. On the other hand, VR projection systems allow one user or a group of users, surrounded with screens, experiencing and sharing the viewpoint of one user in that group. Users stay connected to reference frame of surrounding reality while seeing their own bodies and even each other [16].

\section{VR facilities at TAMUQ}

TAMUQ acquired its first VR system in 2008. At the time, it was a "first of its kind" facility in Qatar, supporting 3D stereoscopic projection which enabled viewers to sense depth within otherwise flat 2D images. The system came with a curved screen (covering 120-degrees of the human field of vision), rear-projected with three 8,000 lumens DLP projectors. The display was edge-blended so as to give the appearance of a single display image with an aggregate resolution of $3576 \times 1024$ pixels (roughly 2.5 million pixels). The mouse and keyboard were the only mode of interaction with the data. Figure 4 shows the facility layout (left) and a visualization of an oil reservoir on the curved screen (right).
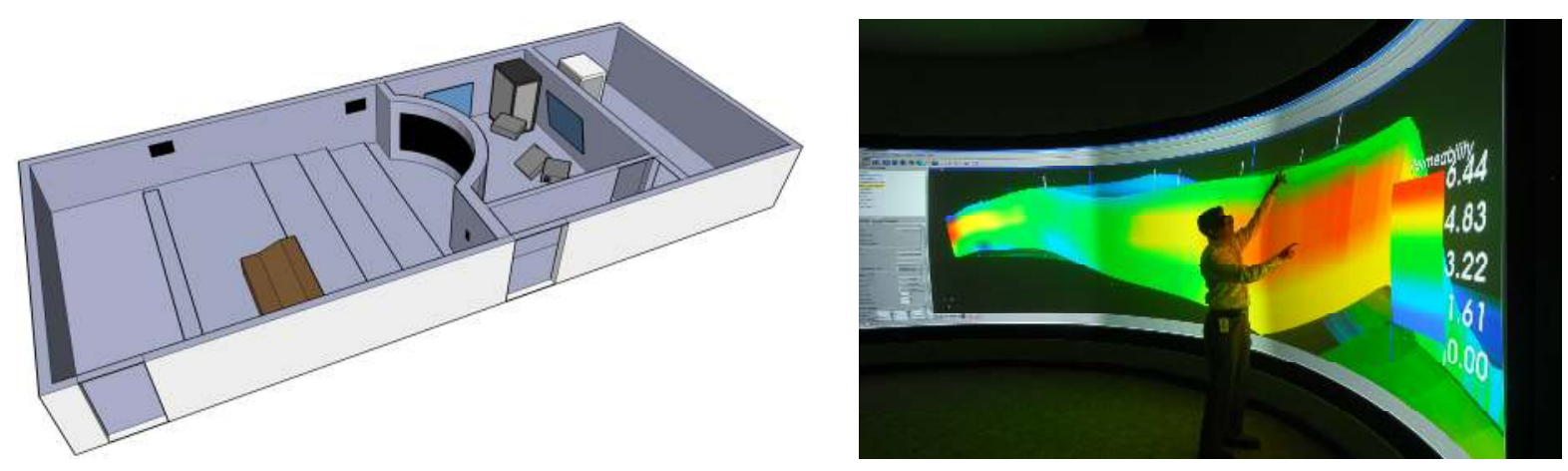

Figure 3. TAMUQ's first curved VR facility layout (left) and real screen (right)

In 2016, TAMUQ upgraded its VR system and replaced the single curved-screen projection system with a four-sided VR space. The new projection system comprises four display surfaces: front, floor and two sides (left and right). The floor display screen increases the degree of immersion and enables even more compelling VR experiences. The side display screens are configurable (see Figure 4) and can be opened completely to make an ultra-wide power-wall, or closed to provide a 
fully immersive experience to viewers. The viewer wears a pair of VR glasses that provide a sense of depth in the images. The images are generated by computers in coordination with a motion tracking system that records the viewer's head position and orientation with respect to the projection surfaces. These head movements are factored in to how the rendered images are displayed on the projection surfaces. As a result, the viewer is able to explore her virtual world by moving around the space and even grabbing objects with a flystick, a wand-like device. Multiple viewers often share virtual experiences and easily carry on discussions, enabling them to exchange insights. One user is considered the active viewer, controlling the stereo projection reference point, while several additional users can simultaneously participate as passive viewers.
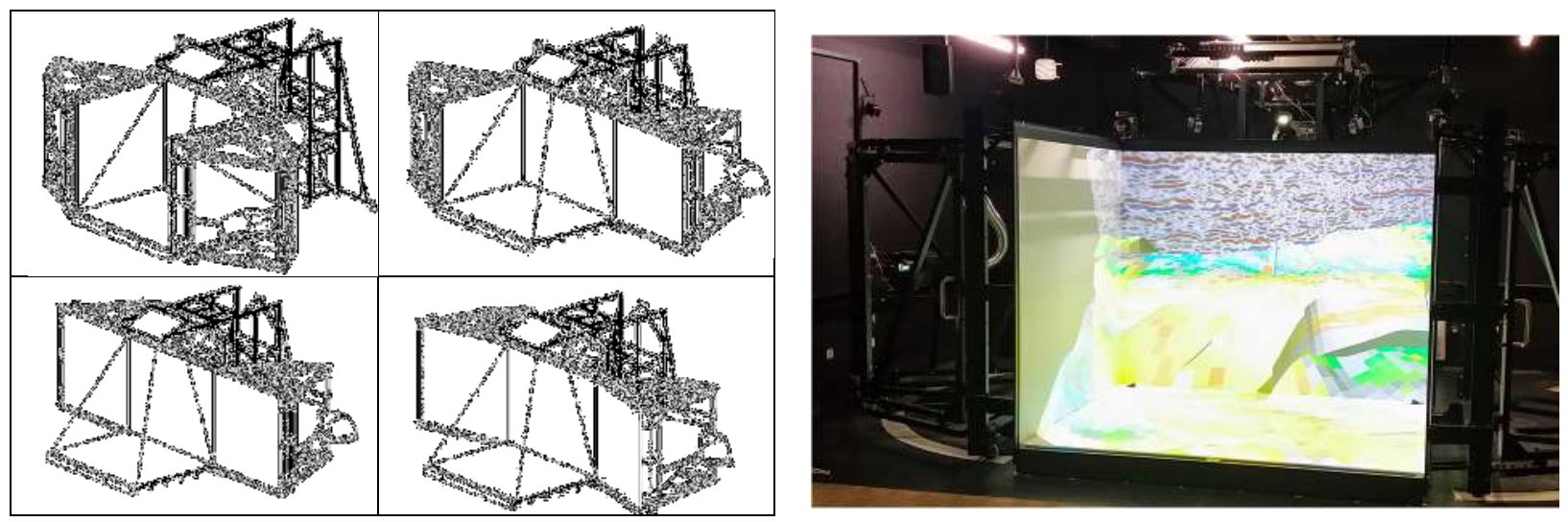

Figure 4. TAMUQ's CAVE VR system configurations (left) and actual picture (right)

In addition to the traditional CAVE VR system, TAMUQ also hosts a number of modern VR devices such as Oculus Rift ${ }^{\circledR}$, HTC Vive ${ }^{\circledR}$, Windows Mixed Reality headsets etc.

\section{Selected VR Projects}

\subsection{Visualization of Petroleum Reservoir Recovery Processes}

The main objective of this project was to provide students a better understanding and insight of fluid displacements in petroleum reservoirs during primary and secondary recovery processes. The project revolves around utilizing the CAVE VR system for the Capstone design course (PETE400). In the project for this course, students developed reservoir simulation models and run simulations to decide on optimum field development strategy. The motivation was to let student interactively change some of the well locations/trajectory to tap into bypassed reserves and immediately see in VR how that changes the overall displacement and recovery. Figure 5 shows an example of an oil and gas reservoir. 


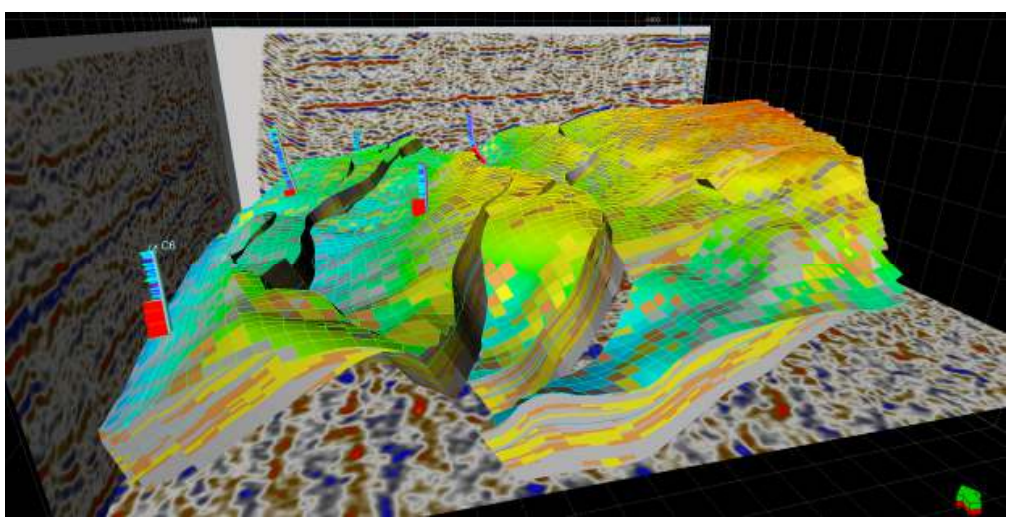

Figure 5. Oil and gas reservoir

The CAVE provided an ideal environment for display of the 3-dimensional reservoir volume and how reservoir pressure and oil saturation change in different parts of this volume with time as the reservoir is depleted. More details about this project can be found in [9].

\subsection{Virtual field trip to drilling rig}

This project was aimed towards incorporating the virtual reality in the petroleum engineering courses. A virtual field trip to an oil drilling rig was developed in VR. The goal was to teach students about the oil and gas facilities which is an essential part of the petroleum engineering curriculum. Instructors often rely on the images/videos of real facilities to demonstrate their operations but that becomes a challenging task especially for complex offshore facilities that are usually very complex and difficult to understand without visiting physically.

Our intention was not to abandon the actual field trips but to improve the learning experience by bringing the "virtual" field trips using the modern, cutting-edge technology in the classroom. The VR makes students feel as protagonists while studying 3D models that enhance their learning experience and increase their engagement. Another motivation comes from the use of such innovative teaching methods in courses such as ENGR 111 can get students interested in the petroleum engineering and potentially increase the enrollment in the program. Other courses that benefited from this project were PETE321, PETE325, PETE402, PETE404, GEOL401, and GEOL404 [17]. The figure below shows some screenshots of the VR experience. 

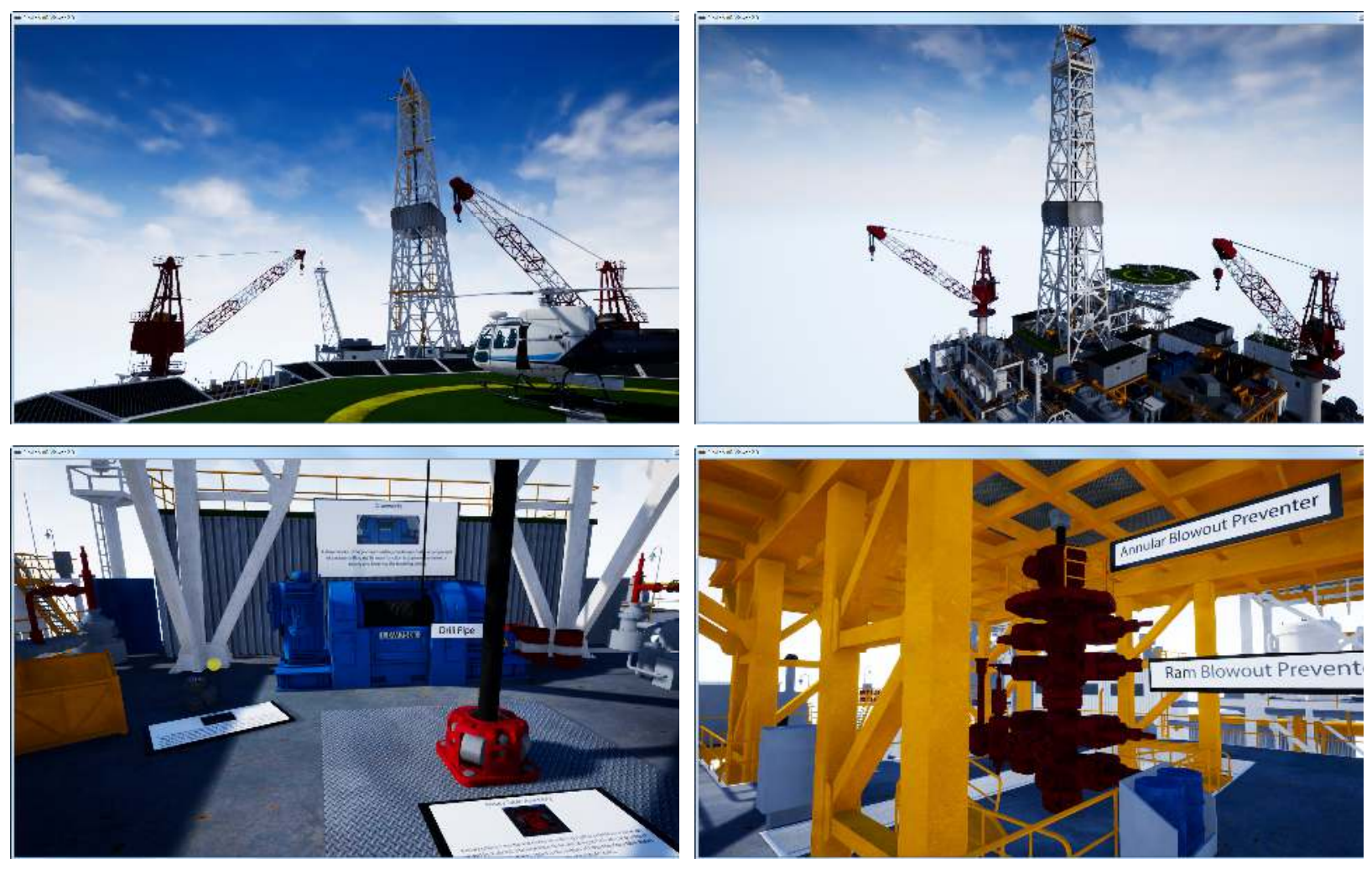

Figure 6. Screenshots of the virtual field trip VR application

\subsection{Digital outcrop study}

The digital outcrop study was a series of projects aiming at utilizing the CAVE VR system in conjunction with the CT scanner to enhance the ability to teach the Petroleum Engineering. The first project was a long-term study of the capillary pressure and imbibition process in the reservoir rock. It involved allowing reservoir cores to imbibe brine water over time. As time passed, the brine would continue to enter the core and move up the interior of the rock, as shown in the figure below. The experiment lasted nearly 10 days and required a large number of scans of the samples. Figure 7 shows the flow of brine water in the reservoir rock at different timesteps after 3D reconstruction using developed applications. The use of a real reservoir rock in the experiment instead of using a capillary tube which while easy to work with is not the same as a true reservoir rock.
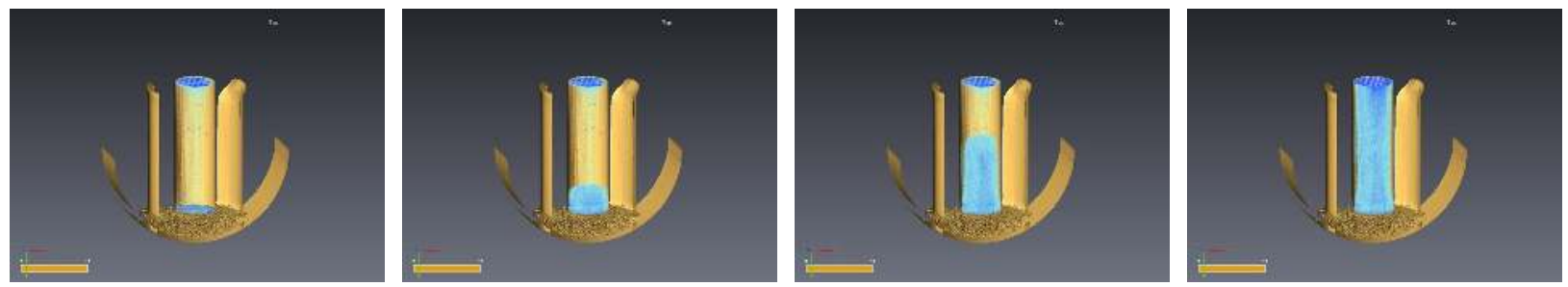

Figure 7. Movement of brine water in reservoir rock 
In the second project of the digital outcrop study, one experiment, the relative permeability experiment, was conducted in the lab taught in PETE 311 course. Each student lab group conducted this experiment and had their results viewed and studied in the CAVE. This latter allowed them to "fly into their samples" and see what their results are first hand and verify them generally by analog methods. Figure 8, for instance, shows the porosity network (left) and cross-section (right) of an outcrop rock. The third project in this study was an extension of the second project, in which students experimented the waterflooding on a reservoir sample for the course PETE 323.
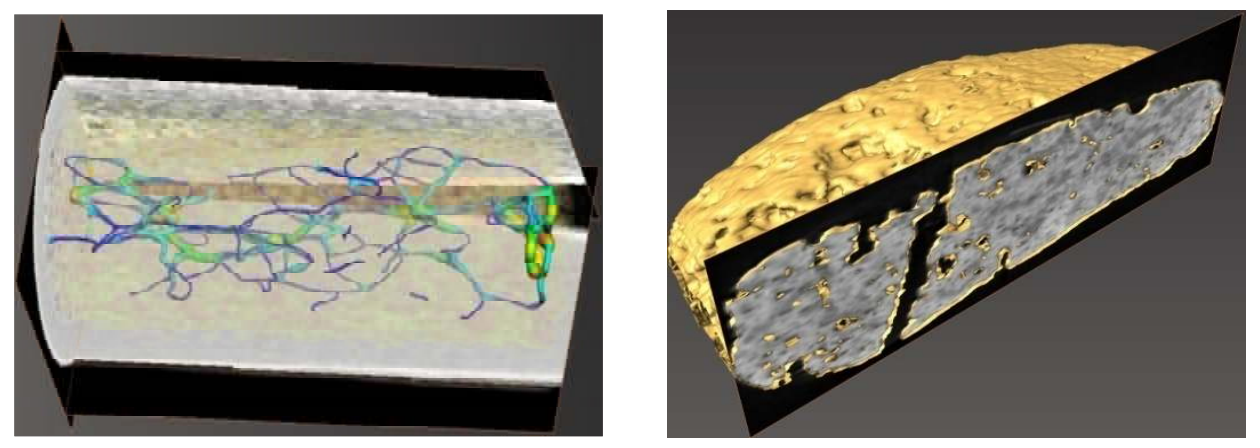

Figure 8. Porosity network (left) and cross-section of an outcrop

\subsection{The virtual museum}

This project was a student-led initiative in which they learned about programming of the 3D graphics in a VR environment and 3D scanning of the objects. They developed a virtual museum to able to catalog and display $3 \mathrm{D}$ replicas of actual artifacts. The intent was to allow visitors the ability to interact with virtual museum pieces by zooming and rotating their 3D renderings, and to have access to relevant textual information about the objects within the display. Figure 9 shows one of the scanned artifacts (left) and a screenshot of the virtual museum VR application (right).

The work of creating the virtual museum pieces progressed through the following workflow:

(1) The object to be modeled was selected.

(2) The selected object was photographed multiple times using a traditional digital camera, each time from a different perspective, where these perspectives were generated by rotating the object by several degrees between pictures, but otherwise leaving the relative positions of the object and camera unchanged.

(3) A software package called "3D SOM Pro" [18] was employed to stitch the multiple photographs into one image, and also to automatically generate a 3D geometry for it. The single image was applied as a texture by the software over the object geometry.

(4) Using the Vizard software package [16], a 3D environment was created for the virtual museum, placing the scanned and modeled objects on displays at selected locations within the museum.

(5) Finally, relevant textual information about each object was inserted into this virtual environment and associated with each object. 

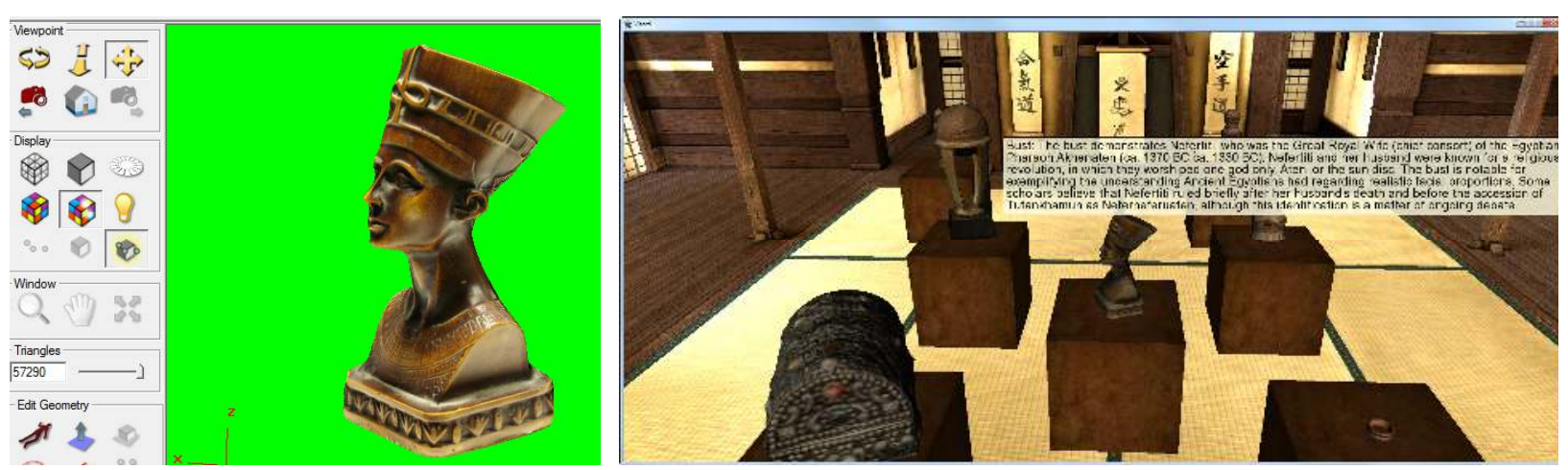

Figure 9. Scanned 3D artifact in 3DSOM software (left) and screenshot of the VR application (right)

\section{Summary and future work}

It appears quite clearly that HMDs and CAVE VR technologies are two different tools allowing a set of uses that only partially overlap. These are still nowadays two distinctive tools meant for a distinguished set of uses. However, we think that we can greatly improve the learning experience if both of these technologies are used at the same time. We could use the CAVE for the lecture style instructions in the classroom and HMDs for the personalized learning experience. For example, an instructor can use the CAVE to take students on a virtual field trip to a remote location such as a chemical plant, where he would teach them about different parts and processes in the facility. Later, students can use the HMDs to train themselves on different tasks at their own pace and convenient. In addition to the VR, we are also exploring the use of augmented and mixed reality in the education.

\section{References}

[1] L. Jensen and F. Konradsen, "A review of the use of virtual reality head-mounted displays in education and training," Education and Information Technologies, vol. 23, no. 4, pp. 1515-1529, 2018.

[2] Oculus, “Oculus rift," 2018. [Online]. Available: http://www. oculusvr. com/rift.

[3] A. Bonasio, "Report: VR and AR Device Market to hit \$1.8 billion in 2018," 9 April 2018. [Online]. Available: http://techtrends.tech/tech-trends/report-vr-and-ar-device-market-tohit-1-8-billion-in-2018/. [Accessed 30 August 2018].

[4] HTC, "Discover Virtual Reality Beyond Imagination," 30 AUGUST 2018. [Online]. Available: https://www.vive.com/.

[5] V. S. Pantelidis, "Reasons to Use Virtual Reality in Education and Training Courses and a Model to Determine When to Use Virtual Reality," Themes in Science and Technology Education, vol. 2, no. 1-2, pp. 59-70, 2010. 
[6] E. Hu-Au and J. J. Lee, "Virtual reality in education: a tool for learning in the experience age,” International Journal of Innovation in Education, vol. 4, no. 4, pp. 215-226, 2017.

[7] T. A. Mikropoulos, A. Chalkidis, A. Katsikis and A. Emvalotis, "Students' attitudes towards educational virtual environments," Education and Information Technologies, vol. 3, no. 2, pp. 137-148, 1998.

[8] O. Bouhali and A. Sheharyar, "3D stereoscopic visualization as a powerful teaching tool," in Proceeding of the International Forum on Engineering Education, Sharjah, UAE, 2010.

[9] A. Gupta, "Experiences in introducing 3D visualization in petroleum engineering education," in SPE Annual Technical Conference and Exhibition, Society of Petroleum Engineers, 2009.

[10] A. Gupta and M. Poppelreiter, "Innovative Field Camp for Education and IndustryAcademic Collaboration in Geology-Based Reservoir Model Building for Carbonate Reservoirs in the Middle East," in SPE Annual Technical Conference and Exhibition, Society of Petroleum Engineers, 2010.

[11] A. Nasir, A. Sheharyar, M. Z. Shakir, K. Qaraqe and O. Bouhali, “3D visualization to aid engineering education: A case study to visualize the impact of wireless signals on human brain," in Global Engineering Education Conference (EDUCON), IEEE, 2014.

[12] E. Tetlow, R. Cuttler, F. Al-Naimi, A. Sheharyar, O. Bouhali, L. Delaney and J. Adcock, "Landscape visualization, sea-level change, and human occupation in Wādī Debayān, north-western Qatar," in Seminar for Arabian Studies , 2013.

[13] A. Abdalla, D. Jayakrishna, N. Ozalp, O. Bouhali and A. Sheharyar, "Three dimensional visualization of hydrogen and carbon black production in a vortex solar reactor," in 2 nd Annual Undergraduate Research Conference on Applied Computing, Dubai, UAE, 2010.

[14] C. Cruz-Neira, D. J. Sandin and T. A. DeFanti, "Surround-screen projection-based virtual reality: the design and implementation of the CAVE," in Proceedings of the 20th annual conference on Computer graphics and interactive techniques, 1998.

[15] Visbox, "VisCube C4-T4 - CAVE Immersive 3D Display," Visbox, [Online]. Available: http://www.visbox.com/products/cave/viscube-c4-t4/. [Accessed 30 August 2018].

[16] WorldViz, "Vizard," WorldViz, [Online]. Available: http://www.worldviz.com/vizard. [Accessed 30 August 2018].

[17] "Texas A\&M University at Qatar | Petroleum Engineering," [Online]. Available: https://pete.qatar.tamu.edu/. [Accessed 5 September 2018].

[18] 3DSOM, “3DSOM - 3D Models from Photos," [Online]. Available: http://www.3dsom.com. [Accessed 30 August 2018]. 